\title{
Resposta da assembleia de formigas na interface solo-serapilheira a um gradiente de alteração ambiental
}

\author{
Diego S. Gomes ${ }^{1}$, Fábio S. Almeida ${ }^{2}$ André B. $\operatorname{Vargas}^{3} \&$ Jarbas M. Queiroz ${ }^{1}$ \\ 1. Departamento de Ciências Ambientais, Instituto de Florestas, UFRRJ. Rod. BR-465, Km 07, 23890-000, Seropédica, RJ, Brasil. (dyegouss@yahoo.com.br; jarquiz@gmail.com) \\ 2. Instituto Três Rios, Departamento de Ciências Administrativas e do Ambiente, UFRRJ. Av. Prefeito Alberto da Silva Lavinas, 1847, 25802-100, Três Rios, RJ, Brasil. (fbio_almeida@yahoo.com.br, \\ autor para contato) \\ 3. Centro Universitário de Volta Redonda - UniFOA. (andrebvargas@yahoo.com.br)
}

RESUMO. O estudo objetivou avaliar a resposta da assembleia de formigas que nidificam na interface solo-serapilheira a um gradiente de alteração ambiental. O estudo foi realizado em três ambientes com diferentes usos do solo: fragmento florestal, sistema agroflorestal (SAF) e pastagem situados em região de domínio da mata atlântica no sudeste do Brasil. Em cada ambiente foram demarcadas 10 parcelas de $1 \mathrm{~m}^{2}$ e todos os ninhos de formigas encontrados foram registrados. Foram encontrados 215 ninhos (103 no fragmento florestal, 64 no sistema agroflorestal e 48 na pastagem), de 31 espécies. O número acumulado de espécies foi maior no fragmento florestal que na pastagem, mas não diferiu do SAF. A composição de espécies foi diferente entre o fragmento florestal e os outros dois ambientes. A riqueza média de espécies de formigas por parcela foi maior no fragmento florestal e menor na pastagem. A quantidade de serapilheira não foi um fator importante para as diferenças na riqueza de espécies. Atividades humanas que reduzam a heterogeneidade da vegetação alteram a composição e diminuem a riqueza de espécies das assembleias de formigas.

PALAVRAS-CHAVE. Agroecossistema, biodiversidade, fatores bióticos.

\begin{abstract}
Response of the ant assemblage to a gradient of environmental change in the soil-litter interface. The present study aimed at assessing the response of the assemblage of ants that nest in the soil-litter interface to a gradient of environmental change. Fieldwork was carried out in three environments under different land use regimes: a forest fragment, an agroforestry system (AFS), and a pasture, all located within the Atlantic Forest of southeastern Brazil. In each environment we marked ten 1- $\mathrm{m}^{2}$ plots and recorded all ant nests found within them. We recorded 215 nests (103 in the forest, 64 in the AFS, and 48 in the pasture) of 31 species. The cumulative number of species was higher in the forest than in the pasture; the forest and the AFS differed from the pasture, but did not differ from each other. Species composition differed between the forest and the other two environments. The average species richness was high in the forest and low in the pasture. The amount of litter did not explain the differences in species richness among environments. Human activities that reduce vegetation heterogeneity change the composition and reduce the richness of the ant assemblage.
\end{abstract}

KEYWORDS. Agroecosystem, biodiversity, biotic factors.

A estrutura das comunidades de formigas tende a variar de acordo com o ambiente em que são encontradas (Folgarait, 1998; Silva \& Silvestre, 2004; RiosCASANOva et al., 2006; Dias et al., 2008). Esse é o caso, por exemplo, das comunidades que vivem na interface solo-serapilheira, que são as mais sensíveis ao tipo de ambiente (SCHMIDT et al., 2013). Isso acontece porque algumas espécies são muito restritivas quanto ao local para nidificação (SOARES \& SCHOEREDER, 2001), outras são intolerantes a ambientes abertos ou muito sombreados (Schmidt et al., 2013). As espécies que são muito restritivas em relação aos recursos e/ou condições do ambiente costumam ser as mais afetadas pelas alterações no ambiente original (LEAL et al., 2012).

A comunidade de formigas da interface solo-serapilheira é diversa taxonomicamente e funcionalmente (HöLlDOBLER \& WILSON, 1990). Seus ninhos podem ser encontrados dentro de frutos, galhos, sementes, entre folhas caídas sobre o solo e no interior do próprio solo (HERBERS, 1989; KaSPARI, 1993; Carvalho \& Vasconcelos, 2002; Almeida et al., 2007). O hábito alimentar é variado também, pois as espécies podem alimentar-se de pequenos animais vivos ou mortos, frutos e sementes de plantas e há o grupo especializado, representado pela tribo Attini, que utiliza diversos elementos de origem vegetal para o cultivo do fungo que as serve de alimento (KASPARI, 2000). Sendo assim, a serapilheira é um elemento importante do nicho ecológico, que fornece abrigo e alimento para muitas espécies de formigas.

A composição e a estrutura da vegetação influenciam diretamente a quantidade e qualidade da serapilheira (Pereira et al., 2007; SANTANA et al., 2009). Por isso, ecossistemas com maior diversidade vegetal tendem a apresentar maior abundância de ninhos e riqueza de espécies de formigas devido, principalmente, à maior quantidade e variedade na oferta de recursos alimentares e de locais para nidificação (DiEHL et al., 2005; Pereira et al., 2007; VArgas et al., 2007; Martins et al., 2011). A substituição de ecossistemas naturais por sistemas agrícolas convencionais ou pastagens ocasiona a simplificação desses ambientes e gera impactos negativos sobre a mirmecofauna (PERFECTO \& Snelling, 1995; Martins et al., 2011). Por outro lado, agroecossistemas mais diversificados, como aqueles que combinam o cultivo de espécies arbóreas e agrícolas, são estruturalmente mais heterogêneos e costumam favorecer a ocorrência de um maior número de espécies (Armbrecht \& Perfecto, 2003; QueIroz et al., 2006).

Estudos que investiguem as características das 
comunidades de formigas em ambientes com diferentes tipos de vegetação se tornam relevantes por identificarem padrões de mudanças nas comunidades frente às alterações ambientais (SchmidT et al., 2013). O uso de formigas como bioindicadores tem sido amplamente disseminado nos últimos anos (e.g. RiBAS et al., 2012), mas a grande maioria dos estudos é baseada no uso de armadilhas para coleta dos organismos. Como toda técnica de coleta é seletiva de alguma maneira, os padrões encontrados são dependentes da metodologia de estudo empregada (Longino et al., 2002). Além disso, a metodologia com uso de armadilhas não proporciona informação sobre o uso de recursos pelas espécies. Isso pode ser conseguido com uma metodologia que envolva a inspeção de espaços delimitados e a coleta manual das espécies (SoARES \& Schoereder, 2001, Pereira et al., 2007).

A partir disso, objetiva-se determinar a resposta da assembleia de formigas a um gradiente de alteração ambiental representado por um fragmento florestal, um sistema agroflorestal e uma pastagem. Para isso foram testadas as seguintes hipóteses: (a) a riqueza de espécies e abundância de ninhos é maior no fragmento florestal, seguido do SAF e da pastagem; (b) a composição de espécies do fragmento florestal difere de forma mais intensa da pastagem do que do SAF e (c) a quantidade de serapilheira é o principal mecanismo que explica o padrão testado acima.

\section{MATERIAL E MÉTODOS}

O estudo foi realizado no Sistema Integrado de Produção Agroecológica (SIPA) (22 45'14"S, $43^{\circ} 40^{\prime} 25^{\prime}$ 'W), Seropédica, estado do Rio de Janeiro. O SIPA tem uma área circular de $1 \mathrm{~km}$ de raio em que, aproximadamente, $2,03 \%$ (6,39 ha) são áreas cultivadas, $54,2 \%(170,40$ ha) áreas de pastagens, $23 \%(73,10 \mathrm{ha})$ fragmentos florestais e a área restante apresenta construções. O clima da região é tropical com inverno seco e verão úmido, com precipitação anual de aproximadamente $1.200 \mathrm{~mm}$ e temperatura média anual de $22,7^{\circ} \mathrm{C}$ (FIDERJ, 1976).

A coleta dos dados foi realizada de agosto a setembro de 2008 em três diferentes ambientes: uma pastagem com alta predominância de Paspalum sp., utilizada para a criação de bovinos $(6,10$ ha); um sistema agroflorestal (SAF) de cultivo de café (Coffea canephora Pierre) (0,43 ha) implantado em 1998 e sombreado por Gliricidia sepium (Jacq.) Steud., uma leguminosa arbórea; e um fragmento de Floresta Ombrófila Densa ( 8 ha) que está em estágio intermediário de sucessão secundária. A distância entre a pastagem e o fragmento florestal é de $300 \mathrm{~m}$. O SAF está entre os dois outros ambientes, cerca de $150 \mathrm{~m}$ de cada um deles.

Para a amostragem dos ninhos empregou-se a metodologia de SOARES \& SCHOEREDER (2001), delimitando-se 10 parcelas de $1 \mathrm{~m}^{2} \mathrm{em}$ cada ambiente, demarcadas com estacas de madeira identificadas, onde ocorreram buscas por ninhos de formigas. A busca por ninhos, em cada parcela, se encerrou quando toda a serapilheira e solo foram averiguados. As parcelas foram alocadas na região central de cada ambiente e a 15 metros de distância entre si. A profundidade de serapilheira foi medida por meio de uma régua graduada em quatro pontos dentro de cada parcela.

Houve procura criteriosa dos ninhos presentes no solo e na serapilheira. Galhos e sementes tiveram seu interior inspecionado para verificar a ocorrência de ninhos e foram anotados os tipos de sítios onde os mesmos foram encontrados (galho, entre folhas, entre cascas de plantas, solo e fruto). Para reduzir possíveis erros quanto à contagem repetida de ninhos de uma mesma espécie em uma mesma parcela, foram realizados testes para avaliar se pertenciam a ninhos distintos. Dessa forma, operárias de cada olheiro foram coletadas e colocadas em contato em uma placa de Petri de 35 x $10 \mathrm{~mm}$. Comportamentos agressivos foram usados como evidência de que operárias pertenciam a colônias diferentes.

Foram coletados espécimes de cada ninho e, quando possível, de castas diferentes; os exemplares foram armazenados em frascos com álcool 70\% devidamente etiquetados. O material foi identificado seguindo Bolton (1994) e Bolton et al. (2006). Quando possível, realizou-se a identificação em nível de espécie, utilizando chaves de revisões taxonômicas e por meio de comparações com as espécimes depositados na Coleção Ângelo Moreira da Costa Lima (CECL) do Instituto de Biologia da UFRRJ.

Para comparar a riqueza total de espécies de formigas entre os ambientes foram geradas curvas de rarefação baseadas no número de registro de espécies (Mao-Tau) através do programa EstimateS 8.0 (Colwell, 2006). Nesse caso, a riqueza de espécies de dois ambientes foi considerada significativamente diferente quando não houve sobreposição entre o intervalo de confiança das curvas de acumulação das mesmas, a um mesmo número de ocorrências.

Para testar se existe diferença na riqueza de formigas ou no número de ninhos por parcela, foram utilizadas ANCOVAs, tendo o número de espécies ou de ninhos por parcela amostrada como variáveis resposta, os ambientes como fatores e a profundidade de serapilheira como covariável (CAMPos et al., 2008). Estas análises foram realizadas utilizando-se o programa estatístico PAST (HAMMER et al., 2003).

Para analisar a influência dos ambientes na estrutura da assembleia de formigas seguiu-se a abordagem de SCHMIDT et al. (2013), com pequenas modificações. Com o auxílio do programa PAST (HAMmer et al., 2003), procedeu-se uma ordenação multidimensional não-métrica (NMDS) utilizando o índice de similaridade de Bray-Curtis e uma ANOSIM para testar a significância das diferenças nas composições 
em espécies dos ambientes estudados. Posteriormente foi conduzida uma análise de SIMPER para quantificar a contribuição de cada espécie para as diferenças de composição entre os ambientes (HAMMER et al., 2003).

Adicionalmente, para testar a relação entre quantidade de recursos - medida pela profundidade de serapilheira -, e a riqueza de espécies e abundância de ninhos de formigas, procedeu-se análises de regressão linear, tendo a profundidade de serapilheira como variável independente e a riqueza de espécies ou abundância de ninhos como variáveis dependentes.

\section{RESULTADOS}

Foram registrados 215 ninhos e 31 espécies de formigas na amostragem. A maioria dos ninhos foi encontrada no solo $(86,5 \%$ do total). Seis espécies se mostraram generalistas quanto aos locais de nidificação, apresentando ninhos na serapilheira e no solo. Por outro lado, quatro espécies encontradas no fragmento florestal nidificaram somente em galhos (Tab. I). Dos ninhos construídos nos componentes da serapilheira, $86,2 \%$ foram registrados no fragmento florestal e 13,8\% no SAF. Na pastagem não foram verificados ninhos na serapilheira. Os galhos foram os componentes da serapilheira mais utilizados para nidificação, representando 79,3\% dos ninhos.

Em relação à riqueza total, o número acumulado de espécies foi maior no fragmento florestal que na pastagem. O SAF acumulou mais espécies que a pastagem, mas não diferiu do fragmento florestal (Fig. 1). O número médio de espécies por parcela foi significativamente maior no fragmento florestal do que na pastagem (ANCOVA, $\mathrm{F}_{2,30}=3,592 ; \mathrm{P}=0,042$; Fig. 2), independente da profundidade da serapilheira $\left(\mathrm{F}_{1,30}=1,724 ; \mathrm{P}=0,201\right)$. Por outro lado, o número de ninhos por parcela não diferiu estatisticamente entre os três ambientes (ANCOVA, $\mathrm{F}_{2,30}=2,874 ; \mathrm{P}=0,075$ ),

Tab. I. Lista de espécies de formigas, abundância e local de nidificação em ordem decrescente de contribuição para as diferenças entre ambientes, segundo análise SIMPER, no Sistema Integrado de Produção Agroecológica (SIPA), Seropédica, RJ.

\begin{tabular}{|c|c|c|c|c|c|c|c|c|c|c|c|}
\hline \multirow[b]{2}{*}{ Taxon } & \multicolumn{2}{|c|}{ Análise SIMPER } & \multicolumn{3}{|c|}{$\begin{array}{c}\text { Abundância média por } \\
\text { ambiente }\end{array}$} & \multicolumn{4}{|c|}{ Locais de Nidificação } & \multirow[b]{2}{*}{ Solo } & \multirow[b]{2}{*}{ Tota } \\
\hline & Contribuição & $\begin{array}{c}\% \\
\text { acumulada }\end{array}$ & SAF & $\begin{array}{c}\text { Fragmento } \\
\text { Florestal }\end{array}$ & Pastagem & Galho & Folha & Fruto & Cascas & & \\
\hline Pheidole sp. 1 & 13,3 & 15,7 & 0,7 & 2,4 & 0,9 & - & - & - & - & 40 & 40 \\
\hline Solenopsis sp. 1 gr. geminata & 12,4 & 30,3 & 1,5 & 0 & 1,5 & - & - & - & - & 30 & 30 \\
\hline Brachymyrmex sp. 1 & 12,2 & 44,7 & 2 & 0,3 & 1 & 1 & - & - & - & 32 & 33 \\
\hline Linepithema neotropicum Wild, 2007 & 6,7 & 52,6 & 0,7 & 0,9 & 0 & - & - & - & - & 16 & 16 \\
\hline Pheidole sp. 4 & 6,3 & 60,1 & 0 & 1,1 & 0,2 & - & - & - & - & 13 & 13 \\
\hline $\begin{array}{l}\text { Linepithema humile angulatum } \\
\text { (Emery, 1894) }\end{array}$ & 5,0 & 65,9 & 0 & 1,7 & 0 & 2 & - & - & - & 15 & 17 \\
\hline Solenopsis sp. 3 & 3,7 & 70,3 & 0 & 1,4 & 0,1 & 10 & 1 & 1 & - & 3 & 15 \\
\hline Pheidole sp. 6 & 2,4 & 73,1 & 0 & 0,1 & 0,3 & - & - & - & - & 4 & 4 \\
\hline Pheidole sp. 2 & 2,3 & 75,9 & 0,4 & 0 & 0 & - & - & - & - & 4 & 4 \\
\hline Pheidole sp. 7 & 2,2 & 78,5 & 0 & 0 & 0,4 & - & - & - & - & 4 & 4 \\
\hline Crematogaster evallans Forel, 1907 & 2,0 & 80,9 & 0,3 & 0 & 0 & - & - & - & 3 & - & 3 \\
\hline Solenopsis sp. 2 & 1,7 & 82,8 & 0,3 & 0 & 0 & - & 1 & - & - & 2 & 3 \\
\hline Hypoponera sp. 1 & 1,6 & 84,8 & 0 & 0,3 & 0 & - & - & - & - & 3 & 3 \\
\hline Camponotus cingulatus (Mayr, 1862) & 1,6 & 86,6 & 0 & 0,4 & 0 & 2 & - & - & - & 2 & 4 \\
\hline Camponotus sp. 1 & 1,3 & 88,2 & 0 & 0,2 & 0 & 2 & - & - & - & - & 2 \\
\hline Cyphomyrmex sp. 1 & 1,3 & 89,8 & 0 & 0 & 0,2 & - & - & - & - & 2 & 2 \\
\hline Brachymyrmex sp. 2 & 1,3 & 91,3 & 0 & 0,4 & 0 & - & - & - & - & 4 & 4 \\
\hline Nylanderia sp. & 0,8 & 92,2 & 0,1 & 0 & 0 & - & - & - & - & 1 & 1 \\
\hline $\begin{array}{l}\text { Gnamptogenys aff. striatula } \\
\text { Mayr, } 1884\end{array}$ & 0,8 & 93,1 & 0 & 0,2 & 0 & 1 & - & - & - & 1 & 2 \\
\hline Wasmannia sp. 1 & 0,7 & 93,8 & 0 & 0,3 & 0 & 3 & - & - & - & - & 3 \\
\hline Monomorium sp. 1 & 0,6 & 94,6 & 0 & 0 & 0,1 & - & - & - & - & 1 & 1 \\
\hline Atta sexdens rubropilosa Forel, 1908 & 0,6 & 95,3 & 0 & 0 & 0,1 & - & - & - & - & 1 & 1 \\
\hline Camponotus rufipes Fabricius, 1775 & 0,6 & 96,0 & 0 & 0,1 & 0 & 1 & - & - & - & - & 1 \\
\hline Mycocepurus smithii (Forel, 1893) & 0,5 & 96,6 & 0,1 & 0 & 0 & - & - & - & - & 1 & 1 \\
\hline Pheidole sp. 3 & 0,5 & 97,2 & 0,1 & 0 & 0 & - & - & - & - & 1 & 1 \\
\hline Oxyepoecus sp. 1 & 0,5 & 97,8 & 0 & 0,1 & 0 & - & - & - & - & 1 & 1 \\
\hline Labidus coecus (Latreille, 1802) & 0,5 & 98,4 & 0,1 & 0 & 0 & - & - & - & - & 1 & 1 \\
\hline $\begin{array}{l}\text { Odontomachus haematodus } \\
\text { (Linnaeus, 1758) }\end{array}$ & 0,5 & 98,9 & 0,1 & 0 & 0 & - & - & - & - & 1 & 1 \\
\hline Pheidole sp. 5 & 0,4 & 99,4 & 0 & 0,2 & 0 & - & - & - & - & 2 & 2 \\
\hline Pyramica eggersi (Emery, 1890) & 0,3 & 99,7 & 0 & 0,1 & 0 & 1 & - & - & - & - & 1 \\
\hline Pachycondyla striata F. Smith, 1858 & 0,3 & 100 & 0 & 0,1 & 0 & - & - & - & - & 1 & 1 \\
\hline
\end{tabular}


sendo que também não houve efeito da profundidade de serapilheira sobre o número de ninhos $\left(\mathrm{F}_{1,30}=0,261 ; \mathrm{P}=\right.$ 0,614; Fig. 2).

Ao se considerar todos os locais ao mesmo tempo, a composição de espécies foi significativamente diferente entre os três ambientes estudados (ANOSIM, $\mathrm{R}=0,2808 ; \mathrm{P}=0,001) . \mathrm{Na}$ comparação par a par, a composição do fragmento florestal diferiu do SAF (R $=0,4937 ; \mathrm{P}=0,001)$ e da pastagem $(\mathrm{R}=0,2856 ; \mathrm{P}=$ $0,003)$, mas não houve diferença entre SAF e pastagem $(\mathrm{R}=0,093 ; \mathrm{P}=0$ 0,092) (Fig. 3). Apenas Pheidole sp.1 e Brachymyrmex sp.1, espécies com maior número de ninhos, foram registradas nos três ambientes estudados. Entre as quatro espécies que mais contribuíram para as diferenças entre os três ambientes, Solenopsis sp.1 gr. geminata e Brachymyrmex sp.1 predominaram na pastagem e no SAF, enquanto Pheidole sp.1 e Linepithema neotropicum foram as mais abundantes, principalmente no fragmento florestal (Tab. I).

A quantidade de serapilheira, medida pela sua profundidade, não influenciou significativamente $\mathrm{o}$ número de espécies (análise de regressão linear, $\mathrm{F}_{1,30}=$ 0,$03 ; \mathrm{P}=0,96$ ) ou de ninhos (análise de regressão linear, $\left.\mathrm{F}_{1,30}=0,56 ; \mathrm{P}=0,459\right)$ por parcela.

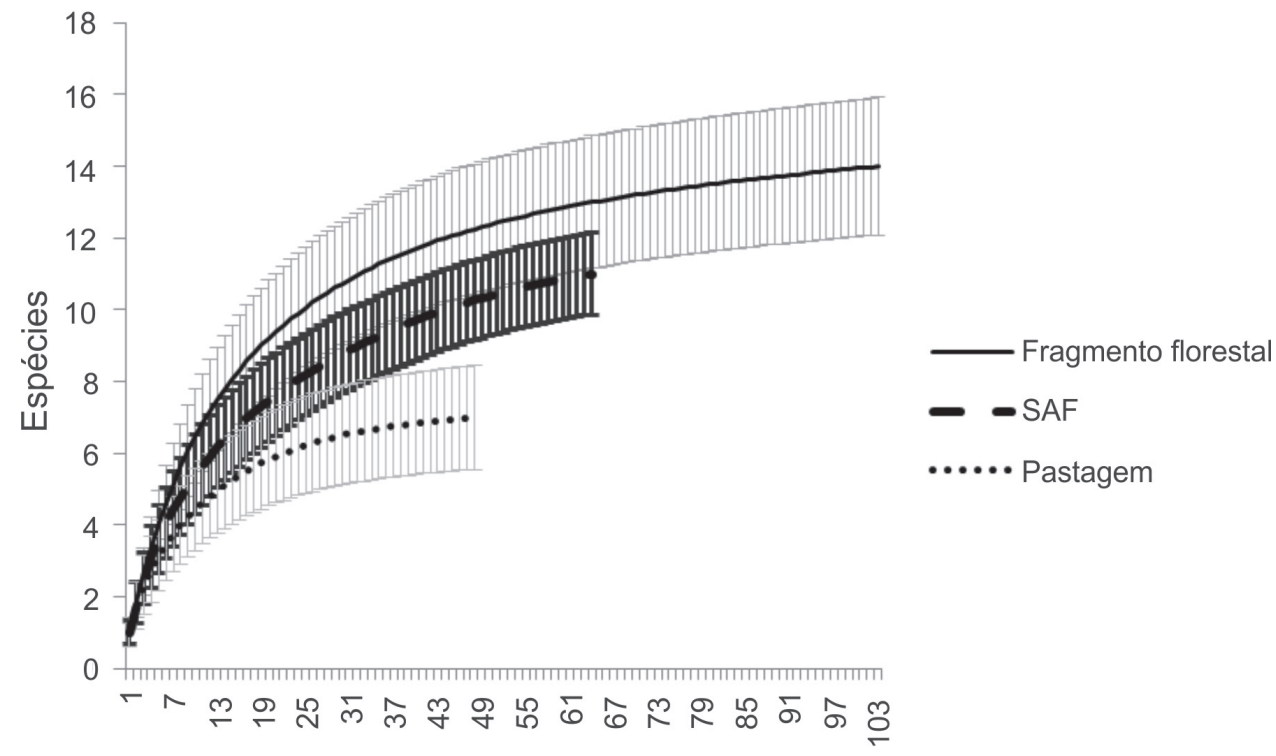

Ocorrências

Fig. 1. Curvas de rarefação de espécies de formigas em diferentes ambientes amostrados em Seropédica, RJ. O eixo y representa a riqueza observada por rarefação pelo método Mao Tau e o eixo x o número de registros (ocorrências) de espécies. As curvas superiores e inferiores para cada ambiente representam o intervalo de confiança de $95 \%$.

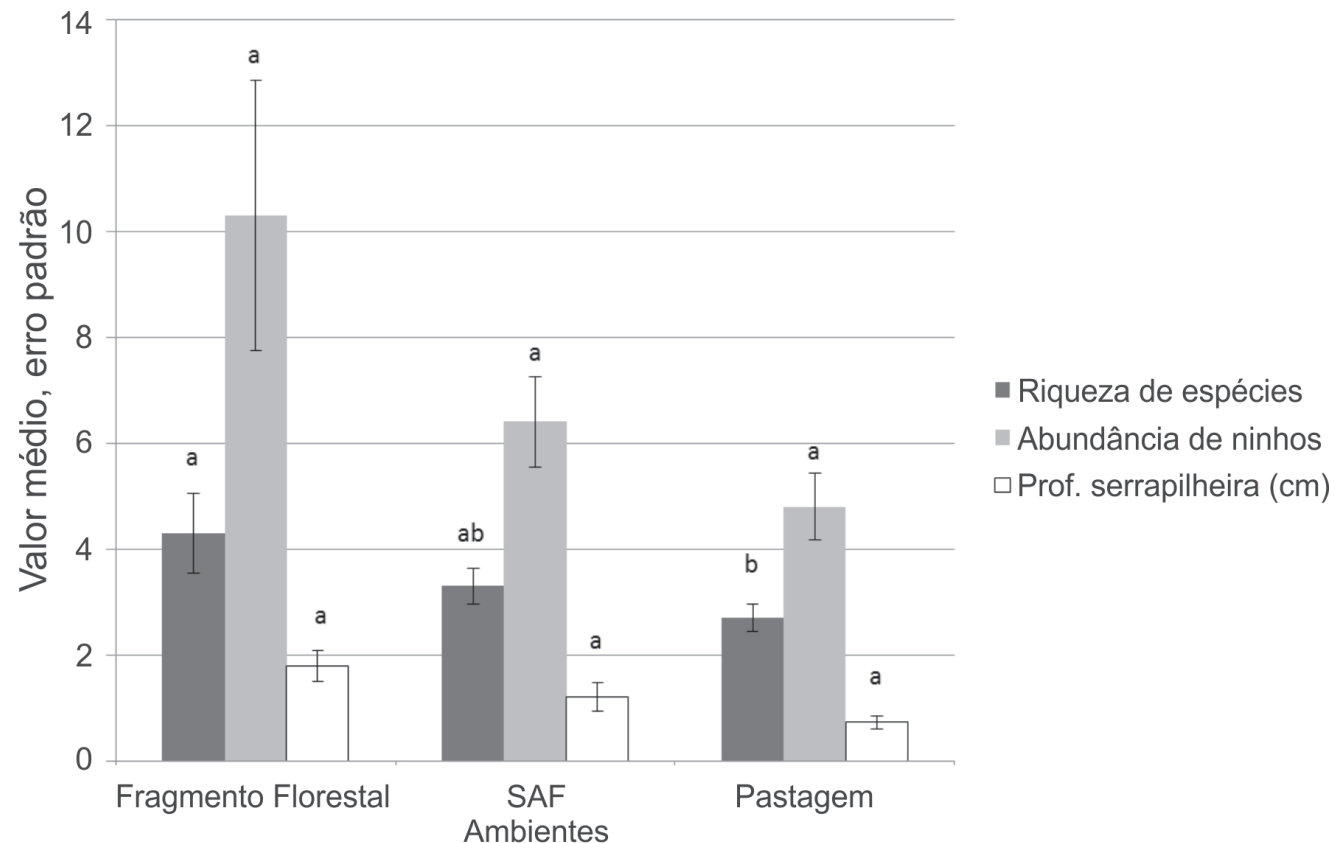

Fig. 2. Valores médios ( \pm erro padrão) para riqueza de espécies, abundância de ninhos e profundidade da serapilheira nos três ambientes amostrados em Seropédica, RJ. Letras diferentes para a mesma variável indicam diferença significativa entre os ambientes $(\mathrm{P}<0,05)$. 


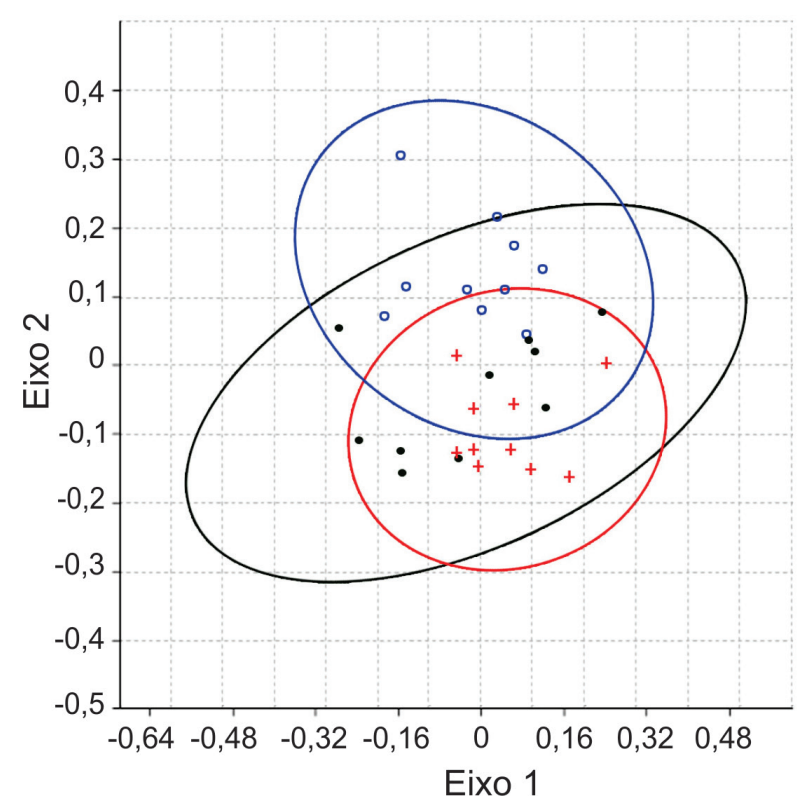

Fig. 3. Configuração bidimensional da ordenação NMDS, realizada com os dados de registros das espécies, por parcela, nos três ambientes do Sistema Integrado de Produção Agroecológica, Seropédica, RJ utilizando a medida de Bray-Curtis (Stress $=0,3029)$ : $(\circ)$ fragmento florestal, (+) SAF e $(\bullet)$ Pastagem.

\section{DISCUSSÃO}

As diferenças na riqueza de espécies entre os ambientes amostrados podem estar relacionadas ao fato de que as práticas agrícolas geralmente reduzem a diversidade de formigas através de alterações nas condições microclimáticas, na oferta de recursos alimentares e de locais de nidificação para as espécies (LOBRY-DE-BRUYN, 1999). Além disso, o fragmento florestal deve ser espacialmente mais heterogêneo por tratar-se de uma floresta secundária em estágio intermediário de regeneração. Comparado com ambientes cultivados, com uma ou poucas espécies vegetais, o fragmento florestal possui condições mais favoráveis para a ocorrência de formigas especialistas em nidificar na serapilheira, o que contribuiu para o aumento na riqueza de espécies (Ribas et al., 2003). Embora a maioria das espécies de formigas tenha sido encontrada nidificando no solo, as diferenças na riqueza entre os ambientes pode ser atribuída, em parte, à presença de espécies que nidificavam na serapilheira do fragmento florestal. Em geral, ambientes com maior diversidade e abundância de espécies vegetais, como o fragmento florestal deste estudo, acabam por disponibilizar maior quantidade e qualidade de recursos para as formigas (VARGaS et al., 2007; Dias et al., 2008). A existência de uma maior variedade de tipos de galhos utilizados pelas formigas para nidificação tem sido apontada como fator importante para a manutenção de alta diversidade de espécies na serapilheira (ARMBrecht et al., 2004; Pereira et al., 2007).

Em relação aos dois manejos agrícolas do solo, $\mathrm{SAF}$ e pastagem, o primeiro foi superior à pastagem para a conservação de uma maior riqueza. Vários autores já comentaram que o cultivo de café sombreado aumenta a diversidade de recursos para a mirmecofauna, possibilitando a manutenção um número mais elevado de espécies (Рerfecto et al., 1996; Armbrecht \& Perfecto, 2003; Perfecto et al., 2003). Além disso, o manejo orgânico - sem a utilização de pesticidas - no SAF também pode ter contribuído para a maior riqueza de espécies em relação à pastagem (DiAs et al., 2008).

A diferença na composição de espécies de formigas entre o fragmento florestal e os demais ambientes pode ser explicada em parte pela maior densidade e riqueza de espécies de árvores (Ribas et al., 2003; LeAL et al., 2012). Tanto na pastagem quanto no SAF as formigas nidificavam predominantemente no solo, mas no fragmento florestal algumas espécies foram encontradas nidificando na serapilheira. A baixa similaridade entre o fragmento florestal e o SAF deve estar ligada à ausência no SAF das formigas que nidificam na serapilheira e ou devido à diferença no sombreamento das duas áreas. No $\mathrm{SAF}$, como na pastagem, deve haver um predomínio de espécies de áreas abertas, enquanto no fragmento florestal predominam espécies de áreas fechadas (SCHMidT et al., 2013).

A relação esperada entre a profundidade da serapilheira e a riqueza de espécies ou abundância de ninhos, como verificada em outros estudos (e.g. VARGAS et al., 2007), não ocorreu. Alguns autores demonstraram que a diversidade dos componentes da serapilheira, e.g. folhas, galhos, flores e frutos, pode afetar mais a diversidade de formigas do que a profundidade da serapilheira em si (ARmBrecht et al., 2004; Pereira et al., 2007). Infelizmente a diversidade de componentes de serapilheira não foi medida no presente estudo.

As práticas agrícolas que reduzem a heterogeneidade da vegetação influenciamnegativamente a riqueza de espécies de formigas e ainda a composição das assembleias, favorecendo espécies mais generalistas de áreas abertas. Assim, a simplificação mais extrema do ambiente, observada em pastagens, é prejudicial para a conservação da biodiversidade de formigas. Os cultivos agrícolas podem prejudicar menos a fauna desde que sejam mais diversificados, como é o caso dos sistemas agroflorestais. As florestas nativas são essenciais para a conservação de formigas, por serem espacialmente mais heterogêneas, ofertando maior variedade de recursos e condições ambientais para as espécies.

A relação entre a estrutura das assembleias de formigas e o tipo de ambiente já foi abordada em trabalhos que utilizaram armadilhas para amostragem das formigas (e.g. Schmidt et al., 2013). Neste estudo, ao invés de armadilhas, foi realizada uma inspeção do solo e dos componentes da serapilheira em parcelas alocadas em diferentes ambientes, para contagem e identificação dos ninhos das espécies de formigas encontradas em um espaço delimitado. Apesar da técnica exigir maior disponibilidade de tempo, o que acaba 
comprometendo o número de amostras possíveis em determinados estudos, uma vantagem é que ela permite avaliar a associação das diferentes espécies com seu uso do ambiente para nidificação, o que facilitou a análise dos padrões encontrados.

Agradecimentos. Aos revisores anônimos e à Rafael Dias Loyola pelas sugestões em uma versão prévia do manuscrito. À EMBRAPA-Agrobiologia, pela autorização de pesquisa no Sistema Integrado de Produção Agroecológica. DSG (Proc. E-26/150.902/2007) e JMQ (Proc.101.472/2010) agradecem à FAPERJ pelo apoio financeiro.

\section{REFERÊNCIAS BIBLIOGRÁFICAS}

Almeida, F. S.; Queiroz, J. M. \& MaYhé-Nunes, A. J. 2007. Distribuição e abundância de ninhos de Solenopsis invicta Buren (Hymenoptera: Formicidae) em um agroecossistema diversificado sob manejo orgânico. Floresta e Ambiente 14(1):33-43.

Armbrecht, I. \& Perfecto, I. 2003. Litter-twig dwelling ant species richness and predation potential within a forest fragment and neighboring coffee plantations of contrasting habitat quality in Mexico. Agriculture, Ecosystems and Environment 97(1):107-115.

Armbrecht, I.; Perfecto, I. \& Vandermeer, J. 2004. Enigmatic biodiversity correlations: ant diversity responds to diverse resources. Science 304:284-286.

Bolton, B. 1994. Identification guide to the ant genera of the world. Cambridge, Harvard University Press. 222p.

Bolton, B.; Alpert, G.; WARD, P. S. \& NASKReCKi, P. 2006. Bolton's Catalogue of Ants of the World. Massachusetts, Harvard University Press (CD-ROM).

Campos, R. I.; Lopes, C. T.; Magalhães, W. C. S. \& Vasconcelos, H. L. 2008. Estratificação vertical de formigas em Cerrado strictu sensu no Parque Estadual da Serra de Caldas Novas, Goiás, Brasil. Iheringia, Série Zoologia 98(3):311-316.

Carvalho, S. K. \& Vasconcelos, L. H. 2002. Comunidade de formigas que nidificam em pequenos galhos da serapilheira em floresta da Amazônia Central, Brasil. Revista Brasileira de Entomologia 46(2):115-121.

Colwell, R. K. 2006. EstimateS: Statistical estimation of species richness and shared species from samples. Version 8.0. Disponível em: $<$ http://viceroy.eeb.uconn.edu/estimates $>$. Acesso em: 12.2008 .

Dias, N. S.; Zanetti, R.; Santos, M. S.; Louzada, J. \& Delabie, J. 2008. Interação de fragmentos florestais com agroecossistemas adjacentes de café e pastagem: respostas das comunidades de formigas (Hymenoptera, Formicidae). Iheringia, Série Zoologia 98(1): 136-142.

Diehl, E.; Sacchett, F. \& Albuquerque, E. Z. 2005. Riqueza de formigas de solo na praia da Pedreira, Parque Estadual de Itapuã, Viamão, RS, Brasil. Revista Brasileira de Entomologia 49(4):552-556.

FIDERJ. 1976. Indicadores climatológicos: sistema de informação para o planejamento estadual. Rio de Janeiro: FIDERJ/ SECPLAN. 54p.

Folgarait, P. F. 1998. Ant biodiversity and its relationship to ecosystem functioning: a review. Biodiversity and Conservation 7(9): 1221-1244

Hammer, O.; Harper, D. A. T. \& Ryan, P. D. 2003. Paleontological Statistics - PAST. Disponível em: <http://folk.uio.no/ohammer/ past>. Acesso em: 16.07.2009.

Herbers, J. M. 1989. Community structure in north temperate ants: temporal and spatial variation. Oecologia 81:201-211.

Hölldobler, B. \& Wilson, E. O. 1990. The Ants. Cambridge, Belknap Press of Harvard University Press. 732p .

KASPARI, M. 1993. Removal of seeds from neotropical frugivore droppings. Oecologia 95(1):81-88.
2000. A primer on ant ecology. In: Agosti, D.; Majer, J. D. Alonso, L. E. \& Schultz, T. R. eds. Ants: standard methods for measuring and monitoring biodiversity. Washington D.C., Smithsonian Institution Press. p. 9-24.

Leal, I. R.; Filgueiras, B. K. C.; Gomes, J. P.; Lannuzzi, L. \& ANDERSEN, A. N. 2012. Effects of habitat fragmentation on an richness and functional composition in Brazilian Atlantic forest. Biodiversity Conservation 21(7):1687-1701.

LOBRY-DE-BRUYN, L. A. 1999. Ants as bioindicators of soil function in rural environments. Agriculture, Ecosystems and Environment 74:425-441.

Longino, J. T.; Coddington, J. \& Colwell, R. K. 2002. The ant fauna of a tropical rain forest: estimating species richness three different ways. Ecology 83:689-702.

Martins, L.; Almeida, F. S.; Mayhé-Nunes, A. J. \& Vargas, A. B. 2011. Efeito da complexidade estrutural do ambiente sobre as comunidades de formigas (Hymenoptera: Formicidae) no município de Resende, RJ, Brasil. Revista Brasileira de Biociências 9(2): 174-179.

Pereira, M. P. S.; Queiroz, J. M.; Souza, G. O. \& Mayhe-Nunes, A. J. 2007. Influência da heterogeneidade da serapilheira sobre as formigas que nidificam em galhos mortos em floresta nativa e plantio de eucalipto. Neotropical Biology and Conservation 2:161-164

Perfecto I. \& Snelling, R. 1995. Biodiversity and the transformation of a tropical agroecosystem: ants in coffee plantations. Ecological Applications 5(4): 1084-1097.

Perfecto, I.; Mas, A.; Dietsch, T. \& Wandermeer, J. 2003. Conservation of biodiversity in coffee agroecosystems: a tri-taxa comparison in southern Mexico. Biodiversity and Conservation 12:1239-1252.

Perfecto, I.; Rice, R. A.; Greenberg, R. \& Van Der Voort, M. E. 1996. Shade coffee: a disappearing refuge for biodiversity. BioScience 46(8):598-608

QueIroz, J. M.; Almeida, F. S. \& Pereira, M. P. S. 2006. Conservação da biodiversidade e o papel das formigas (Hymenoptera: Formicidae) em agroecossistemas. Floresta e Ambiente 13(2):37-45

Ribas, C. R.; Campos, R. B. F.; Schmidt, F. A. \& Solar, R. C. 2012. Ants as indicators in Brazil: a review with suggestions to improve the use of ants in environmental monitoring programs. Psyche 2012:1-23.

Ribas, C. R.; Schoereder, J. H.; Pic, M. \& Soares, S. M. 2003. Tree heterogeneity, resource availability, and larger scale processes regulating arboreal ant species richness. Austral Ecology 28(3):305-314

Rios-Casanova, L.; Valiente-Banuet, A. \& Rico-Gray, V. 2006. Ant diversity and its relationship with vegetation and soil factors in an alluvial fan of the Tehuacán Valley, Mexico. Acta Oecologica 29(3):316-323

Santana, J. A. S.; Vilar, F. C. R.; Souto, P. C. \& Andrade, L. A 2009. Acúmulo de serapilheira em plantios puros e em fragmento de Mata Atlântica na Floresta Nacional de Nísia Floresta - RN. Revista Caatinga 22(3):59-66.

Schmidt, F. A.; Ribas, C. R. \& Schoereder, J. H. 2013. How predictable is the response of ant assemblages to natural forest recovery? Implications for their use as bioindicators. Ecological Indicators 24:158-166.

Silva, R. R. \& Silvestre, R. 2004. Riqueza da fauna de formigas (Hymenoptera: Formicidae) que habita as camadas superficiais do solo em Seara, Santa Catarina. Papéis Avulsos de Zoologia 44(1):1-11.

SoAres, S. M. \& Schoereder, J. H. 2001. Ant-nest distribution in a remnant of tropical rainforest in southeastern Brazil. Insectes Sociaux 48(3): 280-286.

Vargas, A. B.; Mayhé-Nunes, A. J.; Queiroz J. M.; Souza, G. O. \& Ramos, E. F. 2007. Efeitos de fatores ambientais sobre a mirmecofauna em comunidade de restinga no Rio de Janeiro, RJ. Neotropical Entomology 36(1):28-37. 